Revue de droit comparé du travail et de la sécurité sociale

$3 \mid 2020$

La Directive 2019/1158 du 20 juin 2019 concernant l'équilibre entre vie personnelle et vie privée des parents et des aidants

\title{
Développements dans les droits du travail et de
} l'emploi aux Etats-Unis

Risa L. Lieberwitz

\section{OpenEdition \\ Journals}

Édition électronique

URL : https://journals.openedition.org/rdctss/1028

DOI : $10.4000 /$ rdctss. 1028

ISSN : 2262-9815

Éditeur

Centre de droit comparé du travail et de la sécurité sociale

Édition imprimée

Date de publication : 1 novembre 2020

Pagination : 176-179

ISSN : 2117-4350

Référence électronique

Risa L. Lieberwitz, « Développements dans les droits du travail et de l'emploi aux Etats-Unis », Revue de droit comparé du travail et de la sécurité sociale [En ligne], 3 | 2020, mis en ligne le 01 novembre 2021 , consulté le 11 novembre 2021. URL : http://journals.openedition.org/rdctss/1028 ; DOI : https:// doi.org/10.4000/rdctss. 1028

\section{(c) (i) $\odot$}

Revue de droit comparé du travail et de la sécurité sociale est mise à disposition selon les termes de la Licence Creative Commons Attribution - Pas d'Utilisation Commerciale - Pas de Modification 4.0 International. 


\section{RISA L. LIEBERWITZ}

UNIVERSITÉ CORNELL, ECOLE DES RELATIONS INDUSTRIELLES ET DU TRAVAIL

\section{DÉVELOPPEMENTS DANS LES DROITS DU TRAVAIL ET DE L'EMPLOI AUX ETATS-UNIS}

\section{I - COUR SUPRÊME DES ETATS-UNIS}

Dans une décision historique rendue en juin 2020 dans l'affaire Bostock v. Clayton County, Georgia. v. EEOC'1 la Cour suprême des Etats-Unis a estimé que la discrimination fondée sur le sexe, interdite par le titre VII de la loi sur les droits civils de $1964^{2}$, intègre également la discrimination fondée sur l'orientation sexuelle et l'identité de genre. Le titre VII, qui s'applique aux employeurs publics et privés, interdit la discrimination dans l'emploi fondée sur la race, la couleur, le sexe, l'origine nationale et la religion. La décision de la Cour, à 6 voix contre 3, concernait trois affaires regroupées provenant de différentes Cours d'appel fédérales qui avaient abouti à des résultats contradictoires dans l'interprétation du titre VII. II est à noter que deux des juges conservateurs ont rejoint la majorité, dont le juge Gorsuch qui a rédigé l'opinion majoritaire. Adoptant une approche "textuelle », la Cour a ainsi conclu : "Un employeur qui licencie une personne parce qu'elle est homosexuelle ou transgenre la licencie pour des traits ou des actions qu'il n'aurait pas mis en cause pour des personnes d'un sexe différent. Le sexe joue un rôle nécessaire et indiscutable dans la décision, ce qui est interdit par le Titre VII $»^{3}$.

Le 18 juin 2020, la Cour a décidé que l'administration Trump avait agi de manière inappropriée en mettant fin à l'action différée des arrivées d'enfants étrangers (DACA) sans justification suffisante ${ }^{4}$. Créé par l'administration Obama en 2012, le programme DACA protège contre l'expulsion quelques 700000 jeunes immigrés sans papiers qui sont arrivés enfants aux Etats-Unis et ont désormais le droit d'y travailler légalement, d'y poursuivre des études supérieures et d'accéder à d'autres avantages, tels que l'assurance maladie ou le permis de conduire ${ }^{5}$. Dans une décision à 5 voix contre 4, rédigée par le juge en chef Roberts, la Cour a conclu que l'administration Trump avait agi « de façon arbitraire et capricieuse » en ne justifiant pas sa position sur l'illégalité de ne pas expulser, et en ne tenant pas compte des difficultés rencontrées par les bénéficiaires du programme DACA ${ }^{6}$. La Cour a renvoyé l'affaire devant le Département fédéral de la sécurité intérieure (DHS) afin qu'il réexamine ces questions. Lors du renvoi, le DHS a imposé des directives provisoires restreignant la portée du programme

4 Dep't. of Homeland Sec. v. Regents of the Univ. of Cal., _ U.S. _, 140 S. Ct. 1891 (2020).

5140 S. Ct. 1901-02.

6 Ibid., 1913-14. 
$\mathrm{DACA}^{7}$. Des actions en justice visant à contester cette nouvelle orientation ont été engagées ${ }^{8}$.

Dans l'affaire Our Lady of Guadalupe School v. Morrissey-Berru9, la Cour suprême des Etats-Unis a clarifié la portée et l'applicabilité de "l'exception ministérielle » précédemment reconnue par la Cour dans l'affaire Hosanna-Tabor Evangelical Lutheran Church and School v. EEOC ${ }^{10}$. Dans cette affaire, la Cour a jugé que les salariés qui tombent sous le coup de "l'exception ministérielle » n'ont pas le droit de poursuivre les institutions religieuses qui les emploient pour non-respect de la loi contre la discrimination. Si les institutions religieuses sont généralement concernées par les lois sur la discrimination en matière d'emploi, la Cour a en effet estimé qu'elles étaient protégées par la Constitution contre toute ingérence du gouvernement dans leur relation de travail avec leurs salariés " ministres ». Dans l'affaire Our Lady of Guadalupe, la Cour, dans une décision adoptée à 7 voix contre 2, a clairement indiqué que "l'exception ministérielle » favorise largement l'institution religieuse. Dans Hosanna-Tabor, la Cour a refusé « d'adopter une formule rigide pour décider quand on peut considérer un salarié comme un ministre de l'Eglise », mais elle a identifié quatre critères pertinents à prendre en compte. Cependant, dans l'affaire Our Lady of Guadalupe, la Cour n'a pris en compte qu'un seul de ces critères, à savoir le fait que " les responsabilités professionnelles de l'individu impliquent un rôle de transmission du message de l'Eglise et d'exécution de sa mission ${ }^{11}$. Toute autre circonstance, telle que le titre de l'individu ou sa formation religieuse, ne sera prise en considération que dans la mesure où elle éclaire ce critère plus large ${ }^{12}$. Cette approche a conduit la Cour à conclure que les deux plaignants - des instituteurs catholiques chargés d'enseigner différentes matières, dont la religion - relevaient de l'exception ministérielle. Ces enseignants avaient signé des contrats de travail stipulant expressément que leur rôle était de promouvoir la mission religieuse de l'école, et avaient reçu des guides du salarié qui en faisaient état ${ }^{13}$. Ils n'étaient donc pas habilités à engager une action pour discrimination dans l'emploi fondée sur l'âge ou le handicap.

\section{II - COMMISSION NATIONALE DES RELATIONS DE TRAVAIL}

Quatre des cinq sièges de la Commission nationale des relations de travail (la NLRB, ou la Commission) sont actuellement occupés, trois par des membres conservateurs et un par un membre plus libéral. Les trois premiers, majoritaires dans la Commission, ont poursuivi leurs efforts pour restreindre les droits conférés aux travailleurs par la NLRA, soit en annulant, soit en durcissant leurs décisions précédentes. En juin 2020,

7 American Immigration Lawyers Association (26 août 2002) : $\underline{\text { https://www.aila.org/advo-media/aila- }}$ practice-pointers-and-alerts/aila-practice-alert-filing-daca-renewal

8 Bureau du Procureur général de l'Etat de Washington - le procureur général Ferguson - qui actualise le procès DACA (28 août 2020) : https://www.atg.wa.gov/news/news-releases/agferguson-updates-daca-lawsuit-after-trump-administration-again-strips

9 _ U.S. _ 140 S. Ct. 2049 (2020).

10565 U.S. 171 (2012).

11140 S. Ct. 2062-63.

12 Ibid., 2063.

13 Ibid., 2066. 
la NLRB a rendu une décision sur l'applicabilité de la loi nationale sur les relations de travail (NLRA) aux institutions religieuses ${ }^{14}$.

Dans l'affaire Bethany College ${ }^{15}$, la Commission a adopté un critère juridique qui permettra à la quasi-totalité des écoles ou universités religieuses d'échapper à la compétence de la NLRB. En effet, la Commission n'exercera pas sa compétence sur un établissement d'enseignement à but non lucratif qui se présente comme offrant un environnement éducatif religieux et qui est affilié, appartient, est exploité ou contrôlé par une organisation religieuse reconnue ${ }^{16}$. En accord avec la Cour d'appel fédérale qui avait créé ce critère juridique, la Commission a justifié son approche noninterventionniste comme étant un moyen d'éviter l'interdiction constitutionnelle de toute interférence du gouvernement en matière de religion ${ }^{17}$. Cependant, avec cette décision, les professeurs qui enseignent des matières laïques dans ce type de collèges ou d'universités n'auront aucun droit de se syndiquer ou de négocier collectivement en vertu de la NLRA. En adoptant ce critère juridique, la Commission a rejeté le précédent critère qui motivait sa décision de 2014 dans l'affaire Pacific Lutheran University ${ }^{18}$, déclinant l'exercice de sa compétence uniquement lorsque le collège ou l'université considère que les membres du corps professoral jouent un rôle spécifique dans la création ou le maintien de l'environnement éducatif religieux de l'établissement ${ }^{19}$.

La Commission a continué d'élargir le pouvoir de l'employeur et de restreindre le droit à la parole des salariés sur des problèmes rencontrés dans l'entreprise. L'article 7 de la NLRA protège l'action concertée des salariés sur le lieu de travail20, ce qui comprend les discussions entre les salariés, les débats houleux et les critiques acerbes contre les employeurs. En juillet 2020, dans l'affaire General Motors, LLC 21 , la majorité conservatrice de trois membres de la Commission a décidé d'analyser les sanctions appliquées par l'employeur et les licenciements pour cause de propos "insultant », en s'appuyant sur le critère juridique utilisé dans les affaires de discrimination ${ }^{22}$. L'avocat général doit prouver prima facie que les sanctions prises par l'employeur sont motivées par une animosité contre des activités salariales protégées par l'article 7. L'employeur doit alors à son tour prouver qu'il aurait agi de manière strictement identique en l'absence d'une activité protégée par l'article 7.

Ce lien de causalité sera utilisé dans un grand nombre d'affaires, y compris celles relatives à l'action concertée de salariés sur le lieu de travail, sur les réseaux sociaux et pendant une grève ${ }^{23}$. La décision de la Commission dans l'affaire General Motors annule des précédents de longue date qui avaient défini les activités protégées par l'article 7 d'une façon plus large, incluant les discours chargés d'émotion, voire

1429 U.S.C. § 151 et suiv.

15369 NLRB n98 (2020) (résumé de l'arrêt).

16 Ibid., p. 3.

17 Ibid., p. 20.

18361 N.L.R.B.1404 (2014).

$19 \mathrm{lbid}$.

2029 U.S.C. §157.

21369 NLRB nº127 (résumé de l'arrêt).

22 Ibid., p.5.

23 Ibid., p.1. 
insultants ou les « accès de colère » qui font souvent partie des discussions, des débats ou autres actions collectives sur les conditions de travail ${ }^{24}$. La Commission justifie cette nouvelle règle en invoquant la nécessité de permettre aux employeurs de lutter contre le harcèlement au travail, interdit par les lois anti discrimination ${ }^{25}$. Cependant, Mark Pearce, l'ancien président de la NLRB, a noté que la décision de la Commission dans l'affaire General Motors pouvait aussi permettre aux employeurs de sanctionner leurs salariés qui avaient à juste titre « introduit des questions de racisme dans le scénario ${ }^{26}$. De l'avis de Pearce : «Suite aux protestations contre le meurtre de George Floyd, l'attitude de la Commission consiste maintenant à dire que si vous voulez conserver votre emploi, vous feriez mieux d'être soumis, car vous pourriez être licencié si vous osez protester verbalement contre vos conditions de travail exécrables $»^{27}$.

\section{III - DROITS DES SALARIÉS PENDANT LA PANDÉMIE DE COVID-19}

Etant donné la faiblesse de l'Etat providence aux Etats-Unis, I'action du gouvernement s'est révélée cruciale pour fournir des aides d'urgence sous forme d'indemnités de chômage et de congés payés aux millions de personnes licenciées ou susceptibles de l'être - pendant la pandémie de Covid-19. Si le Congrès a adopté une législation d'urgence prévoyant de tels avantages, cette aide gouvernementale est néanmoins restée limitée par leur caractère provisoire (expirant en juillet 2020), et par l'exemption des exigences en matière de congés payés accordée à de nombreux types d'entreprises, par exemple celles employant 500 personnes ou plus ${ }^{28}$.

En raison de l'impasse politique actuelle entre démocrates et républicains à Washington, la nouvelle législation sur les avantages sociaux n'a pas encore été adoptée. La santé et la sécurité au travail ont également été source de sérieuses préoccupations, l'administration fédérale de la santé et de la sécurité au travail (OSHA) ayant fait l'objet de vives critiques pour son incapacité à adopter des règles de sécurité au travail obligatoires, dans un tel contexte ${ }^{29}$. En outre, l'OSHA n'a imposé que des amendes minimes aux grandes entreprises de conditionnement de viande ayant enfreint les règles de sécurité qui ont ainsi provoqué la mort de travailleurs essentiels pendant la pandémie ${ }^{30}$.

\section{Ibid., p.2.}

25 Ibid., p. 26.

26 H. A. Kanu, « NLRB's GM Ruling Gives Employers More Slack to Punish Speech (1) », Bloomberg Law, 21 juillet 2020, p. 2.

27 Ibid.

28 J. Smialek et J. Tankersley, « Economic Data Points to Pause in Recovery as Aid Programs Expire», New York Times, 21 août 2020.

29 Comité de rédaction, "Under Trump, OSHA's Covid-19 Response Is Failing Workers ", New York Times, 14 septembre 2020 .

30 Ibid. 